DRAFT VERSION JANUARY 20, 2020

Preprint typeset using LATEX style emulateapj v. 01/23/15

\title{
ON THE DICHOTOMY BETWEEN NORMAL AND DWARF ELLIPTICALS
}

\author{
JAMES M. SCHOMBerT ${ }^{1}$ \\ ${ }^{1}$ Department of Physics, University of Oregon, Eugene, OR USA 97403 \\ Draft version January 20, 2020
}

\begin{abstract}
Using images from the SDSS DR13 library, we examine the structural properties of 374 bright (classed E0 to E6) and dwarf ellipticals (classed $\mathrm{dE}(\mathrm{nN})$ to $\mathrm{dE}(\mathrm{N})$ ). The sample combines a multicolor sample of bright ellipticals (252 galaxies with $\left.M_{g}<-20\right)$ with a new sample of faint ellipticals (60 galaxies with $M_{g}>-20$ ) which overlaps the dwarf elliptical sample (62 galaxies) in luminosity and size. The faint ellipticals extend the linear structural correlations found for bright ellipticals into parameter space not occupied by dwarf ellipticals indicating a dichotomy exists between the two types. In particular, many faint ellipticals have significantly higher effective surface brightnesses compared to dE's which eliminates any connection at a set stellar mass. Template analysis of the three subsets of ellipticals demonstrates that the bright and faint ellipticals follow the same trends of profile shape (weak homology), but that dwarf ellipticals form a separate and distinct structural class with lower central surface brightnesses and extended isophotal radii.
\end{abstract}

\section{INTRODUCTION}

Their simple morphology and smooth light distribution has made elliptical galaxies the default standard for studying galaxy structure and kinematics. Distinguished in their morphology only by their outer axial ratio, the class of ellipticals display remarkable uniformity in structure parameters as a function of stellar luminosity (a proxy for total stellar mass, see Schombert 2013). For example, ellipticals display a smooth correlation between characteristic scalelength and luminosity, a linear transition in surface brightness profile shape with luminosity and bright ellipticals have higher central stellar densities (i.e., surface brightnesses) with higher luminosities which decreases in a uniform fashion down to total luminosities around $M_{B}=-18$.

However, deep studies of the Virgo cluster suggested a different type of elliptical exists below $M_{B}=-18$, the subclass of dwarf ellipticals (dE, Sandage \& Binggeli 1984). Dwarf ellipticals appeared to be morphologically distinct from normal ellipticals (normal defined as ellipticals with power-lawed profiles, near $r^{1 / 4}$ in shape) and were considered for many years to constituted a separate type of elliptical (Wirth \& Gallagher 1984) by morphologists who claim there is no difficulty in separating normal ellipticals from dwarf ellipticals simply based on their visual appearance (in particular, their diffuseness). Whether this perceived difference can be mapped into quantitative structure parameters was unclear and highly debated (see Graham 2013 for a review), but there is no doubt that bright ellipticals are power-law (i.e., $\mathrm{r}^{1 / 4}$ ) in shape and dwarf ellipticals are closer to exponential (Binggeli, Sandage \& Tarenghi 1984). However, the evolution in profile shape could be a smooth function of luminosity indicating similar formation and evolutionary scenarios for bright and dwarf ellipticals (Jerjen \& Binggeli 1997). And there is no reason to assume that dwarf ellipticals are not 'normal', in the sense of having peculiar structure. They are uniform as a class and we simply use the designation of 'normal' to describe those historically well studied, higher luminosity ellip- ticals. Likewise, we maintain the designation of $\mathrm{dE}$ to describe the dwarf ellipticals rather that the more common dSph classification that extends to luminosities well below this study (Kormendy et al. 2009).

Investigating the connection between dwarf and normal ellipticals has been a challenge due to the fact that normal ellipticals less luminous than $M_{B}=-18$ are rare, but dE types (with luminosities down to -12) are numerous in nearby clusters such as Virgo and Fornax. This has led to a bifurcation in the samples with bright ellipticals observed in the local Universe (distances out to $100 \mathrm{Mpc}$ ) and a large sample of dwarf ellipticals from nearby clusters. If there is a connection, a smooth transition from bright to low luminosity ellipticals, then the nomenclature of 'dwarf' is simply an artificial slice by luminosity. However, if there is a distinct break in the structure of normals to dwarf ellipticals, then this may signal a separate formation process or different evolutionary histories.

Importantly, as pointed out by Graham (2013), the non-linear behavior of correlations between structural parameters and scaling laws extracted from fitting functions, combined with a gap in elliptical samples with respect to luminosity (bright versus dwarf) can result in an apparent dichotomy in the elliptical sequence. While dwarf ellipticals display a wide range in spatial and kinematic properties (Conselice et al. 2001) plus star population characteristics (Poggianti et al. 2001), a continuum with respect to structure would establish a single scenario for most early-type galaxies independent of their mass. Thus, it is critical to fill the gap in luminosity and extend the normal elliptical morphology sample to fainter luminosities with objects that have well defined surface brightness profiles for a direct comparison of structure. That is the goal of this study.

\section{SAMPLE \& DATA REDUCTION}

This study is an outgrow from a study of bright ellipticals tracing colors from the near-UV to the nearIR (Schombert 2016). That sample was selected from the RSA and UGC catalogs to find undisturbed ellipti- 
cals by morphology and isolation from bright stars and other nearby galaxies. For that sample, only $5 \%$ of the galaxies were fainter than $M_{B}=-19$. To extend the elliptical sequence, we have selected 60 more ellipticals from the recent early-type catalog of Dabringhausen \& Fellhauer (2016) specifically for low absolute magnitude. Again, pure elliptical morphology and isolation were the primary criteria, and the target had to be in the SDSS DR13 image library.

In addition to faint ellipticals (classed as E in Dabringhausen \& Fellhauer), 52 dwarf ellipticals were also selected from the dwarf elliptical sample of Lisker, Grebel \& Binggeli (2008) for study (again, isolation was the primary criteria). Of this 52,49 are classed $\mathrm{dE}(\mathrm{N})$, eight are classed $\mathrm{dE}(\mathrm{nN})$ and five as $\mathrm{dE}(\mathrm{bc})$ based on the Lisker scheme. All of these galaxies are in the Virgo cluster with excellent photometry from SDSS. The Virgo sample was combined with a sample of group dE from Dabringhausen $\&$ Fellhauer for a total dwarf sample of 62 galaxies. In addition, we have supplemented the faint elliptical sample with 6 ellipticals from the ACSVCS Virgo sample (Chen et al. 2010) plus 11 dwarf ellipticals from the Chen et al. study. The combined sample (bright, faint and dwarf) contains 374 ellipticals with photometry from SDSS ugri images and the full data (luminosities, colors and structural parameters) will be released in Schombert (2017).

For comparison, we have also extracted the structural parameters for $210 \mathrm{dE}$ 's from Gavazzi et al. (2005), a deep Virgo study with the $2.4 \mathrm{~m}$ Isaac Newton Telescope. We have 42 ellipticals in common with the Chen et al. sample and 27 with Gavazzi et al. , reduced for their surface brightness profiles independently. All the luminosities and structure parameters were within $5 \%$ of the photometric and fitting accuracies. For galaxies in common, we have used our own photometry as we did not have access to the raw surface photometry for the above published studies, and the remaining members of their samples were too faint for SDSS analysis.

Data reduction of the flattened, calibrated images from the SDSS archive was performed with the galaxy photometry package ARCHANGEL (Schombert 2011). These routines, most written in Python, have their origin back to disk galaxy photometry from the late 1980's and blend in with the GASP package from that era (Cawson 1987). The package has four core algorithms that 1) aggressively clean and mask images, 2) fit elliptical isophotes to produce surface photometry, 3) repair masked regions then perform elliptical aperture photometry and 4) determine aperture colors and asymptotic magnitudes from curves of growth and determine accurate errors based on image characteristics, such as the quality of the sky value.

The photometric analysis of ellipticals branches into four areas; 1) isophotal analysis (the shape of the isophotes), 2) surface brightness determination and fitting (2D images reduced to 1D luminosity profiles), 3) aperture luminosities (typically using masked and repaired images and elliptical apertures) and 4) asymptotic or total magnitudes (using curves of growth guided by surface brightness data for the halos, see Schombert 2011). Ellipticals are the simplest galaxies to reduce from 2D images to 1D luminosity profiles since, to first order, they have uniformly elliptical shaped isophotes (Jedrzejewski 1987). Where many ellipticals display disky or
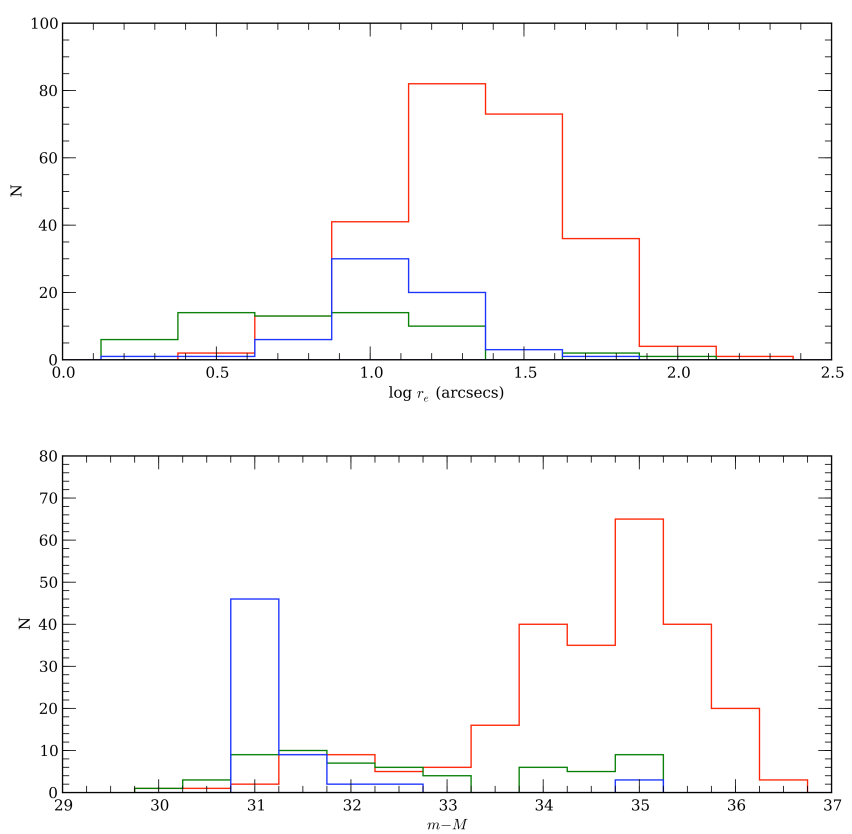

FIG. 1.- Histograms of distance modulus and effective radius in arcsec units. The red line is the bright ellipticals $\left.M_{g}<-20\right)$, green line is faint ellipticals $\left(\mathrm{M}_{g}>-20\right)$ and the blue line is the dwarf elliptical sample. The criteria for isolation avoids many of the nearby rich clusters (e.g., Virgo and Fornax) for the bright elliptical sample with a mean $m-M$ of 34.5. The dE sample is concentrated in the Virgo cluster at $m-M=31.1$ and the faint ellipticals are distributed at various distances in between. The more distant bright elliptical sample means that the scalelength structural parameters (such as effective radius, $r_{e}$ ) are similar in arcsecs to the $\mathrm{dE}$ sample. All the structural parameters are measured to be well outside the radius where PSF effects dominate and at mean surface brightnesses well above the noise limits of the sky brightness (see Schobmert 2017 for a more detailed discussion of the data sample).

boxy isophotal shapes (Kormendy \& Bender 1996), this deviation is at the few percent level and has a negligible effect on the surface brightness profiles, aperture luminosities or colors values. All surface brightness values are determined using the generalized radius $(\sqrt{a b})$ rather than the major axis. Using the major axis is only warranted if there is some confidence that the object is oblate. Dwarf ellipticals follow the same axial ratio distributions as bright ellipticals (Lisker et al. 2007) so an oblate shape is not indicated for that sample as well.

Fits to the surface brightness profiles followed the prescription of Schombert (2013) for both the $r^{1 / 4}$ and Sérsic $r^{1 / n}$ functions. As noted in that study, the value for the Sérsic $n$ index can vary depending on the region of the profile with greater weight (typically the inner with its higher photometric accuracy). As the structural differences between normal and dwarf ellipticals focuses on the behavior of the inner profile shape, we elect to use the inner profile fitting procedure outlined in Schombert (2013). This gives greater weight to the higher surface brightness inner data points and uses photometric accuracy (RMS around each elliptical isophote) to determine the weighting in the outer data points.

Fits were made to extinction corrected profiles. While PSF corrections were applied, all the galaxies were fit 
outside the 1.5 arcsec radius as an additional constraint against PSF errors. Surface brightness errors were determined from RMS errors on each ellipse combined with error due to sky uncertainty. The former dominates the inner isophotes, the later dominates the outer data points. Total magnitudes were determined by two techniques, 1) fits to the curve of growth as an extrapolation to a total magnitude and 2) a $20 \%$ correction to the Kron magnitude determined from the surface brightness profiles (see Schombert 2016). Both techniques yielded the same luminosity to within $2 \%$ for $95 \%$ of the sample. Galaxies with unusual surface brightness profiles, or curves of growth that did not converge, were eliminated from the sample.

All distance related parameters used the CMB distance from NED (for the bright ellipticals) or the distances found in the Dabringhausen \& Fellhauer catalog. For faint, nearby ellipticals, distances were also collected from redshift independent distances found in NED (particularly important for the faintest of the normal ellipticals, see §3). A majority of the dwarf ellipticals are in the Virgo cluster and a distance modulus of 31.09 was assumed for all of them.

The distribution of distance moduli is shown in Figure 1. The isolation criteria produces a sample of bright ellipticals that avoids nearby rich clusters. The dwarf ellipticals are concentrated in the Virgo cluster. In addition, the distribution of effective radius (from Sérsic $r^{1 / n}$ fits) is shown in units of arcsecs to demonstrate that all the structural parameters are determined from regions of the three samples that are similar in resolution element size and well outside the PSF limited requiem. Effective surface brightness for all three samples were also well above the sky noise limits (see Schombert 2017 for a larger discussion of the samples and analysis).

\section{STRUCTURAL RELATIONSHIPS}

Fits with the Sérsic function outputs three parameters, the effective radius $\left(r_{e}\right.$, in $\left.\mathrm{kpc}\right)$, the effective surface brightness $\left(\mu_{e}\right.$, in $g$ mags $\left.\operatorname{arcsec}^{-2}\right)$ and the concentration or shape index $n$. About $80 \%$ of the sample had $n$ values less than 6 , which means that $r_{e}$ is approximately the half-light radius $\left(r_{h}\right)$ and $\mu_{e}$ is the surface brightness at the half-light point (Graham \& Driver 2005). All these structural parameters are correlated with stellar luminosities (a proxy for total baryonic mass) as has been shown by Schombert (2013). Two of these correlations are shown in the top panels of Figure 2.

Graham (2013) divides structural parameter behavior into three types 1) linear relations, 2) non-linear or curved relations and 3) broken relations. Foremost of the linear relations is the luminosity-concentration correlation which is at the heart of the photometric plane (Graham 2002) for the $n$ index can substitute for the velocity dispersion in the usual Fundamental Plane correlations. Whereas Schombert (2013) found the $M_{t}$ versus $\log n$ relation to be less well defined for bright ellipticals, this is due to the degeneracy in the $n$ index for shallow profile slopes typical to ellipticals brighter than -21 . In fact, as can be seen in Figure 2 by comparing the left panels, the $n$ index varies uniformly with luminosity and the new faint elliptical sample connects the dwarf sequence to the bright ellipticals in a smooth, even fashion. The slope for that relationship is consistent with the slope
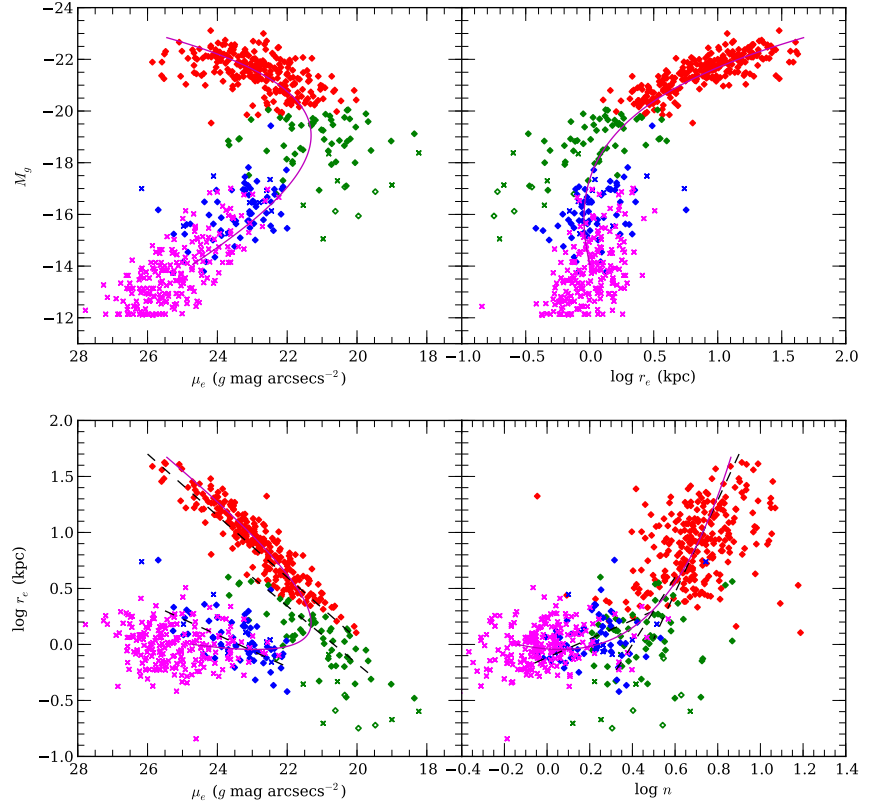

FIG. 2.- Structural parameter space for SDSS $g$ magnitudes and surface brightness fits. The top two panels display total luminosity $\left(M_{g}\right)$ versus Sérsic effective radius $\left(r_{e}\right)$ and surface brightness $\left(\mu_{e}\right)$. The bottom two panels display the relationships between Sérsic parameters $r_{e}, \mu_{e}$ and the concentration index $n$. The normal elliptical sample (morphologically classed as 'E' and having power-law shaped profiles) are divided into bright (red) and faint (green, the Chen et al. data is shown as crosses). The dwarf elliptical sample (morphologically classed as 'dE') are shown as blue symbols, the Gavazzi et al. dE sample are shown as magenta symbols. The curved relationships from Graham \& Guzmán (2003) are shown as magenta lines in each panel. The open green symbols are six faint ellipticals discussed in the text. The black dashed lines are the trends determined from template analysis in $\S 4$ for each of the three subsets.

found by Graham \& Guzmán (2003) for the full sample from dwarf to bright ellipticals.

The relationships comparing effective radius and surface brightness to total luminosity are displayed in the top panels of Figure 2. The total magnitude for the sample is shown on the y-axis, $M_{g}$, plotted against effective radius $\left(r_{e}\right)$ and surface brightness $\left(\mu_{e}\right)$. The sample is divided into three subsets, bright ellipticals (with $M_{g}<-20$, red), the faint ellipticals (green) and the morphologically classified dE's (blue). The faint ellipticals all have "E" classifications, confirmed by visual inspection of their images and comparison of their surface brightness profiles to brighter ellipticals. None have the diffuse appearance that distinguishes the $\mathrm{dE}$ class.

Several well-known historical trends are displayed. The trend of decreasing effective surface luminosity with increasing total luminosity for normal ellipticals is evident. As outlined in Schombert (2013), this reflects the increasing shallowness of the typical elliptical profile slope as the galaxy grows larger and brighter. There is also the obvious size-luminosity relation (Kormendy 2009) for bright ellipticals in the top right panel of increasing effective 
radius $\left(r_{e}\right)$ with elliptical total luminosity.

While both luminosity relations for bright ellipticals (red) are roughly linear, there is significant curvature as anticipated by analysis of Graham \& Guzmán (2003). As pointed out by Graham (2013), the lack of strict homology for ellipticals leads to non-linear relationships between the various structure parameters extracted from Sérsic fits. The curved relationships, as outlined in Graham (2013), are defined from the fact that the central surface brightness, $\mu_{e}$, and the concentration index, $n$, are linearly correlated with total luminosity. One can then derive the expected correlations between effective radius $\left(r_{e}\right)$ and surface brightness $\left(\mu_{e}\right)$ versus total luminosity based on the their coupling within the Sérsic function. This will only apply if all ellipticals are well fit by the Sérsic function, but this is certainly the case if one ignores the complications introduced by core processes which are not relevant to this discussion.

The resulting curved relations from Graham (2013) are shown in Figure 2 as the solid lines. While the correlation between $\mu_{o}$ and $n$ breaks down for the very brightest ellipticals (there is a degeneracy in the fitting process, see Schombert 2013), the curved relations are an excellent description of the behavior of the fitting parameters are a function of total luminosity (top panels). The same curved relations are plotted in the fitting parameter space (bottom panels) which are also in excellent agreement, connecting the bright and dwarf ellipticals. These revised structure correlations are the main argument against a dichotomy between normal and dwarf ellipticals as the curved relation connect the two branches into one branch, a continuum of ellipticals by luminosity that follow the same fitting function (a Sérsic $\mathrm{r}^{1 / n}$ function).

Also, historically, the claim for a dichotomy focused on fitting linear relations to the brightest ellipticals which clearly placed the dwarf ellipticals in a separate part of structural parameter space (Kormendy 1977). For example, the $r_{e}$ versus total luminosity diagram (top right panel in Figure 2, often called the size-luminosity diagram) displays a nearly linear behavior for ellipticals brighter than -19 (the typical luminosity cutoff for a dwarf elliptical). The morphologically classed dwarf ellipticals display larger $r_{e}$ for their luminosity (in agreement with their diffuse appearance) with little indication of a strict correlation with luminosity itself. Other studies have attempted to fit two separate linear relations to the normal and dwarf ellipticals (see Dabringhausen et al. 2008, Lisker 2009), but, in fact, the dE's effective sizes seems uncorrelated (although always between 0.5 and $2 \mathrm{kpcs}$ ) with respect to their total stellar mass, rather than a proper linear relationship. Although it is true that all dE's with $r_{e}$ larger than $1 \mathrm{kpc}$ have a mean $M_{g}$ of -16.5 and the smaller subset has a mean $M_{g}$ of -15.8 , barely significant.

The relationship between $r_{e}$ and $\mu_{e}$ (the Kormendy relation, bottom left panel in Figure 2) displays the most salient characteristic differences between normal and dwarf ellipticals. The trend for fainter effective surface brightness $\left(\mu_{e}\right)$ with increasing size $\left(r_{e}\right)$ is evident. The degree of linearity is questionable but, to first order, the extension of the bright elliptical sequence to fainter luminosities is roughly linear (a statement of the accu- racy of the Sérsic fitting function over a large range in luminosities). The curved relation from Graham (2013) is shown and connects the normal to the dwarf sequence, but at the expense of ignoring the fainter ellipticals. The size-surface brightness relation also predicts nearly constant effective radius $\left(r_{e}\right)$ for the dwarf ellipticals (thus, decreasing surface brightness results in a more diffuse appearance with decreasing luminosity). The trend for this sample is lowest $\mu_{e}$ dwarf ellipticals are slightly larger in $r_{e}$ compared to the brighter dE's. Most importantly, the new sample of fainter normal ellipticals do not follow the curved relationship, although they are well fit by the Sérsic function (see below).

Key to the claim of a dichotomy between normal and dwarf ellipticals are the very faintest normal ellipticals in Figure 2, those with small $r_{e}$ but with high surface brightnesses $\left(\mu_{e}<21 \mathrm{~g}\right.$ mag $\left.\operatorname{arcsecs}^{-2}\right)$. They appear to extend the bright elliptical sequence to smaller $r_{e}$ and brighter $\mu_{e}$ in a roughly linear fashion from the bright ellipticals. In particular, they occupy portions of structural space that are outside the predications from the Graham curved relations that are intended to connect the normal and dwarf sequences. However, Chen et al. (2010) argues that using inaccurate morphological information artificially forces a dichotomy by dividing the elliptical sample exactly where the curved relations connect the bright and faint elliptical sequence. It is certainly true that the number of ellipticals between the top of the $\mathrm{dE}$ sequence (at $M_{g}=-18$ ) and the bottom of the bright elliptical (at $M_{g}=-19$ ) is quite sparse even when combining Chen et al. and our sample (only 34 galaxies not classified as $\mathrm{dE}$ are less then -19 in luminosity). Chen et al. find only four galaxies in higher surface brightness region (marked as green crosses in Figure 2) and conclude that secular processes (such as tidal stripping, Bekki et al. 2001) could have produced their unusual structure in the very rich Virgo environment.

From the comparison with the Chen et al. study, it is clear that any claim of dichotomy is based whether the ellipticals, classified as E, between -19 and -16 in Figure 2 are actually distinct from the dE galaxies of similar luminosity. Chen et al. concludes that this inflection point is due to structural non-homology (presumingly from the homologous dE's to the weak homology found for bright ellipticals). And a lack of dichotomy is supported by the smooth transition in other characteristics from $\mathrm{dE}$ to $\mathrm{E}$ (e.g., color and metallicity).

To examine this interpretation more closely, five of these high surface brightness, faint ellipticals are marked by open symbols in Figure 2 and their individual profiles are shown in Figure 3 (along with a dE of similar luminosity, IC3443). All five are well fit by the Sérsic function with low effective radii $\left(r_{e}\right)$ and bright effective surface brightnesses $\left(\mu_{e}\right)$. All five profiles follow the trend in profile shape displayed by the bright end of the normal elliptical template profiles, meaning they are more power-law shaped than exponential (i.e., $n>1$, see $\S 4$ ).

In addition, their visual morphology is clearly distinct from dwarf ellipticals. Figure 4 displays a low contrast greyscale of a visually classified dwarf elliptical (IC3443, type $\mathrm{dE}(\mathrm{N})$ from Lisker et al. 2008) side-by-side with a faint normal elliptical (VCC1627, whose profile is in Figure 3). Each galaxy was selected to be similar in ellipticity and luminosity. The diffuse appearance to IC3443 


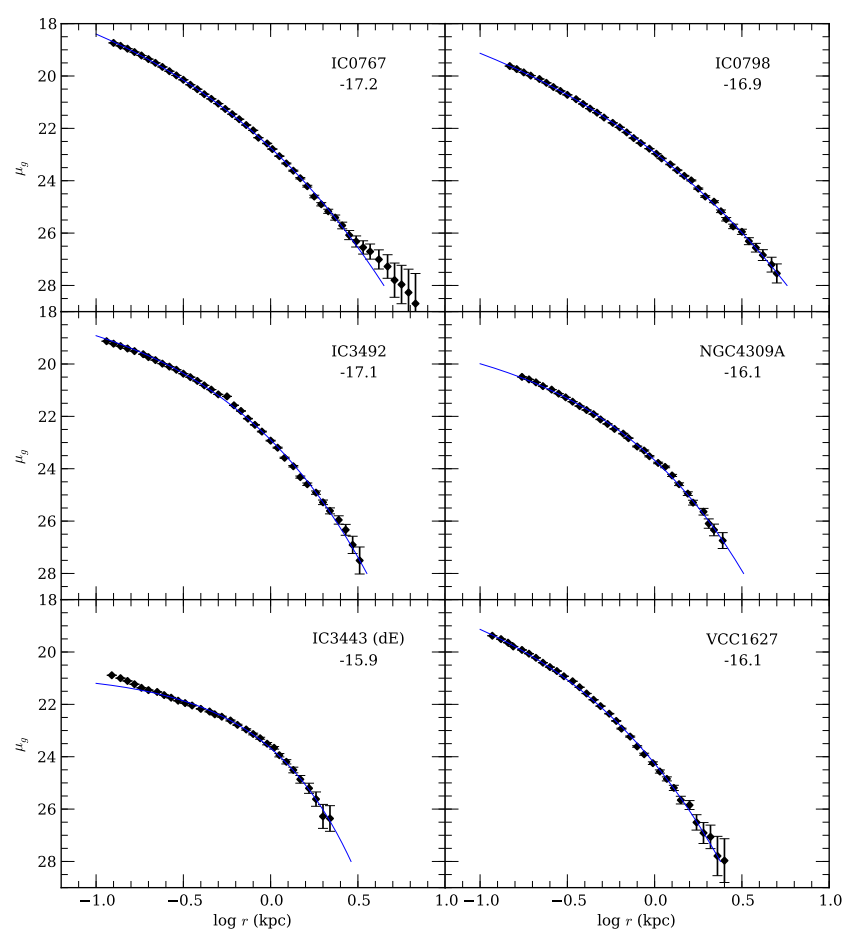

FIG. 3.- Surface brightness profiles for five normal ellipticals with luminosities less than -17.5 (shown as open symbols in Figure 2). Blue lines display their best $\chi^{2}$ Sérsic fits. All six are well fit by the Sérsic function. IC3443 (bottom left panel) is a classic $\mathrm{dE}$ of similar luminosity shown for comparison. Both IC3443 and VCC1627's greyscale images is shown in Figure 4.

is the quality that morphologists use to definite the $\mathrm{dE}$ class and is obvious in the greyscale image (see also, Figure 3 from Wirth \& Gallagher 1984). This distinction is important, as both galaxies have similar stellar masses and the difference relates to individual galaxy's profile slope, where the more shallow profile produces a more diffuse appearance.

Distance errors might account for some of the faint ellipticals deviating from the dwarf elliptical sequence. For example, under estimating their distance would produce larger effective radii $\left(r_{e}\right)$ and move them onto the $\mathrm{dE}$ sequence in the top right panel of Figure 2. However, surface brightness is distance independent (aside from redshift corrections) and no distance errors can move the faintest ellipticals to fainter effective surface brightnesses $\left(\mu_{e}\right)$ in the top left panel of Figure 2.

The faint elliptical region in structure diagrams are known to be populated by the class of elliptials called compact ellipticals (cE). The prototype object being M32 with the largest sample of $\mathrm{cE}$ type ellipticals from the AIMSS project (Norris et al. 2014). Based on their Figures 11 and 14, the AIMSS cE's would overlap the smallest $\left(r_{e}\right.$ between 0.1 and $\left.1 \mathrm{kpc}\right)$ and highest surface brightness ( $\mu_{e}$ greater than 21) faint ellipticals from our sample. The AIMSS survey extends the elliptical sequence beyond the $\mathrm{cE}$ and faint ellipticals to luminosities given by ultra-compact dwarfs (UCD, $M_{g}>-12$ ). Notability, the sequence of $\mathrm{cE}$ and UCD's connects linearly with the brighter ellipticals in terms of scalelength. The relationship between surface brightness of cE's and nor- mal ellipticals increases with decreasing luminosity, but the UCD's decrease in effective surface brightness with decreasing luminosity in the same fashion as the dwarf ellipticals.

Comparison with the $\mathrm{cE}$ sample is problematic as a majority of the AIMSS cE's are embedded in the envelopes of other bright galaxies or in the center of high density clusters making extraction of their surface brightness profiles nearly impossible. For a handful of AIMSS cE's with large enough distances from other galaxies for adequate surface brightness analysis, their luminosity and structure characteristics were identical to the faint ellipticals of our sample with similar luminosities. In particular, their effective surface brightnesses were high and their characteristic scalelengths were low in agreement with their low luminosities.

It has been proposed by many studies (Bekki et al. 2001, Chilingarian et al. 2009, Pfeffer \& Baumgardt 2013) that compact ellipticals are formed by tidal stripping of either brighter ellipticals or disk galaxies (removing the disk and leaving a compact bulge). In addition, tidally induced star formation can increase the stellar densities in the core regions, increasing the inner surface brightness of these objects. Just tidally stripping by itself will not change the shape of the inner surface brightness profile of a bulge or elliptical. However, the effective surface brightness is approximately the luminosity of a galaxy inside its effective radius, divided by the area given by the effective radius. Thus, reducing the effective radius by tidally stripping will increase the effective surface brightness, even if not directly changing the stellar densities in the core regions of a galaxy. Thus, the argument is made that ellipticals occupying the region given by a linear extrapolation of the bright elliptical sequence (high $\mu_{e}$, low $r_{e}$ and intermediate $n$ values) are, in fact, tidally stripped bright ellipticals and not part of the structural sequence from $\mathrm{E}$ to $\mathrm{dE}$ imposed by formation processes.

Of the 62 faint ellipticals in our sample, slightly less than half of them are members of groups or have close companions. Those which have companions, they are all the lesser member of the pair (statistically, this is expected). Tidal effects could strip the outer envelopes of many of these faint ellipticals, reducing $r_{e}$. The key issue is if the profile shape of faintest ellipticals have more in common with the bright elliptical sequence or the dwarf sequence.

We conclude the analysis from structure relations by noted that Graham \& Guzmán (2003) argue that the curved relations demonstrate that the apparent dichotomy is, in fact, due solely to a smooth and steady change in profile shape from bright ellipticals to faint dwarfs. The linear portions of the structure diagrams occur where the concentration index, $n$, is large and effective radius, $r_{e}$, is more strongly correlated with luminosity. The underlying linear correlations are between luminosity and $n$ or $\mu_{e}$, which are more critical in defining a galaxy's profile shape. Thus, there is no dichotomy as the various structure diagrams (with seemingly separate regions of parameter space) are simply reflecting a smooth transition from bright to dwarf ellipticals in profile shape.

However, as noted by Janz \& Lisker (2009), the first order behavior of the various structure diagrams is ex- 

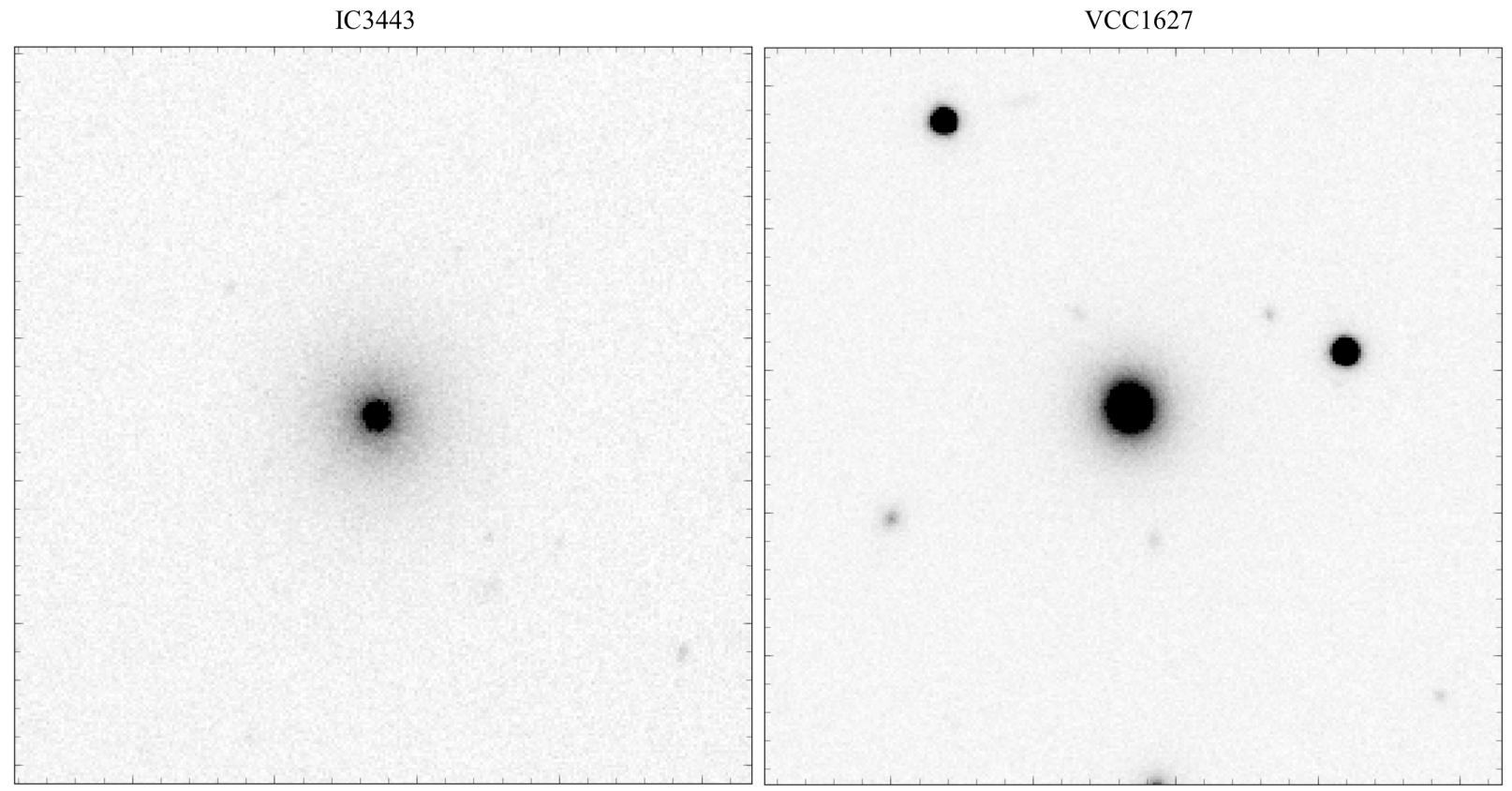

FIG. 4. - SDSS $g$ images for the dE IC3443 $\left(M_{g}=-15.8\right)$ and E class VCC1627 $\left(M_{g}=-16.1\right)$. Each frame is 100 arcsecs on a side (approximately $8 \mathrm{kpc}$ for each galaxy) where the greyscale is set at $23 \mathrm{~g}$ mag arcsecs $^{-2}$ for the blackest level and $26 \mathrm{~g}$ mag $\operatorname{arcsecs}^{-2}$ for the sky level in both frames. The diffuse appearance of IC3443 is the visual signature of the dE class compare to normal ellipticals.

plained by varying profile shapes, but there is a significant number of ellipticals brighter than dE's with smaller effective radius $\left(r_{e}\right)$. As noted above, these objects could be the result of tidal stripping, as proposed for M32 (Bekki et al. 2001), but they conclude that distribution of structural values is much larger than that expected by random scatter and that, in particular, the size-luminosity relation can not be fully explained by a truncated profile shape. A averaged comparison of profile shapes requires the use of template analysis pioneered by Schombert (1987), and will be explored in the next section.

\section{TEMPLATE ANALYSIS}

A clearer view of the elliptical dichotomy can be deduced through the use of template analysis. This technique, first used for brightest cluster ellipticals (Schombert 1987), and expanded for 2MASS ellipticals in Schombert (2013), uses the total surface brightness profile of a galaxy to construct average templates, rather than forcing a fitting function onto a profile shape and then extracting structure parameters from the function best fits. Template comparison is non-parametric and has the advantage of being free of coupling effects between fitting function parameters plus presents a more accurate measure of whether isophotal structure is a smooth function of total galaxy mass (a key test of homology from various galaxy formation scenarios). It has the disadvantage of not reducing a $2 \mathrm{D}$ profile into a few 1D parameters which makes comparison of large samples difficult.

To determine if there is a dichotomy between dwarf and normal ellipticals in terms of structure, we are basically asking if dwarf and normal elliptical surface brightness profiles are self-similar. In other words, are dwarf ellipticals simply scaled down versions of their brighter (i.e. more massive) cousins, as the curved relations from Graham (2013) suggest. The problem with this determination is that normal ellipticals themselves do not display absolute structural homology (Schombert 2015). Their profile shapes do change smoothly with luminosity (or scalelength, so-called weak homology), but they are not self-similar as can be seen in Figure 3 of Schombert (2015). The brightest ellipticals are mostly $\mathrm{r}^{1 / 4}$ in shape, but gradually develop more curvature (a lower Sérsic $n$ index) with decreasing luminosity. Thus, while normal ellipticals are homologous in profile shape within limited luminosity bins, they do not strictly display complete homology.

On the other hand, this uniform change in structure with luminosity can be used for comparison to dwarf ellipticals. For the new sample of faint ellipticals can be compared both to the morphologically classified dwarf ellipticals and the brighter ellipticals with the same template analysis technique. The results from this analysis are shown in Figure 5. As defined in $\S 2$, the normal elliptical sample is divided into two samples, bright and faint with a luminosity cut-off at $M_{g}=-20$ ). The bright sample contains 252 galaxies, the faint sample contains 60 galaxies (green symbols in Figure 2). Template averaging used $1 / 2$ mag bins from -23 to -16 . For comparison, the lowest luminosity template for the 2MASS $J$ templates were $-21.5 \mathrm{~J}$ which corresponds to roughly $-20 \mathrm{~g}$.

The resulting normal elliptical templates agree well with the templates defined in Schombert (2013) using 2MASS $J$ profiles. A similar shift from $\mathrm{r}^{1 / 4}$-like to significantly more curvature with decreasing luminosity is evident (for reference, two pure $\mathrm{r}^{1 / 4}$ profiles are indicated in Figure 5). The faintest luminosity bins (below 


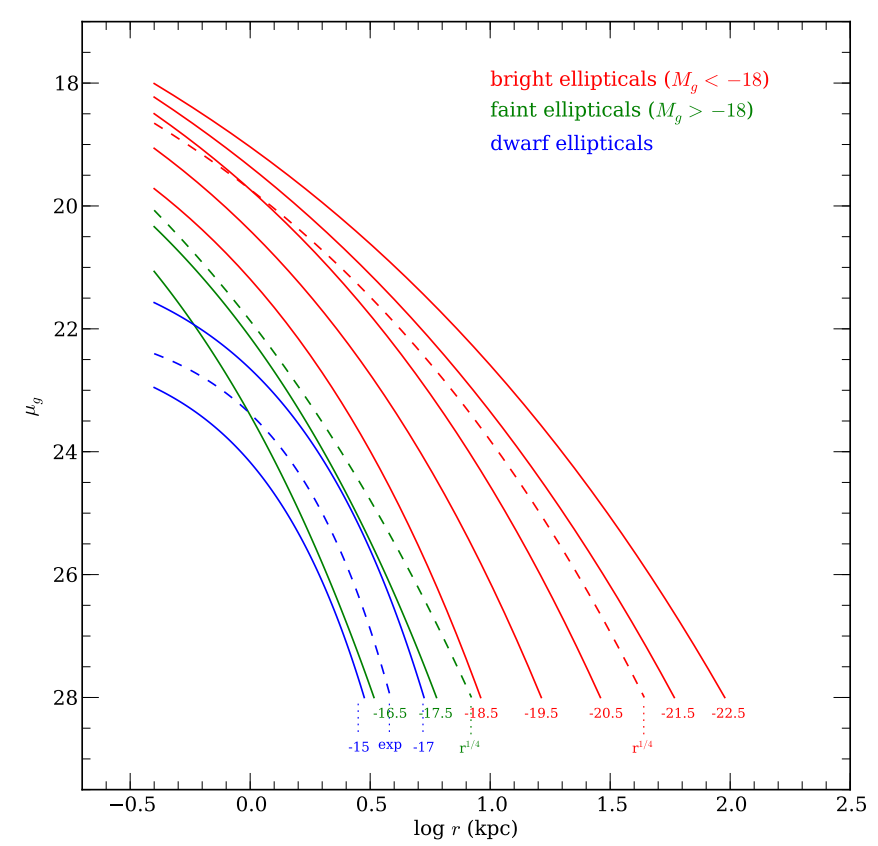

Fig. 5.- Template profiles constructed by the methods outlines in Schombert (2013). The red profiles (parameterized by total magnitude) are for bright ellipticals, and agree with the $V$ templates from Schombert (1984) and the 2MASS $J$ profiles. While the profiles as a function of luminosity are not self-similar (homology), the change with luminosity is smooth and quantifiable. The green templates are constructed from faint normal ellipticals and follow the same profile trend as the bright ellipticals. The templates for dwarf ellipticals are shown in blue and are clearly distinct from the normal elliptical templates with lower central surface brightness and more extended isophotal radii. Reference profiles displaying the $\mathrm{r}^{1 / 4}$ and exponential shape are shown as dashed lines.

-18) are shown in green as these luminosities are equivalent to the luminosities of the dwarf elliptical sample. A luminosity cutoff at -18 seems arbitrary but, in fact, there are very few dwarf ellipticals (by morphology) with higher luminosities. We note that the faintest normal elliptical profiles follow the exact same trend as outlined by the brighter profiles (i.e., they continue the weak homology trends). This would indicate that the continuation of the linear trends in the structure diagrams of Figure 2 are accurate and the normal ellipticals follow a different structural sequence compared to dwarf ellipticals. The Sérsic fits to the templates are shown in Figure 2 as dashed lines.

We perform the same analysis on the 62 dwarf ellipticals in the sample binned into four luminosity bins starting at -14 . Two of the templates $(-15$ and -17$)$ are shown for reference in Figure 5 (blue curves). It is immediately obvious that the $\mathrm{dE}$ templates have a very different shape compared to the normal ellipticals and are not a continuation of the normal elliptical sequence. For a set value of galaxy luminosity (i.e., stellar mass), the $\mathrm{dE}$ templates are lower in central surface brightness, more extended in isophotal radius and display greater curvature (i.e., lower $n$ values) than the normal ellip- ticals. Drawn for reference in Figure 5 is an exponential $(n=1)$ profile (dashed blue line). While the normal ellipticals are nearly $\mathrm{r}^{1 / 4}$ (although deviating systematically at lower luminosities), the dwarf ellipticals are closer to an exponential profile than $r^{1 / 4}$ (something known for many decades, see Caldwell 1983; Binggeli \& Cameron 1991).

Note the $\mathrm{dE}$ templates have greater isophotal radii than normal ellipticals at any luminosity, and are offset by lower central surface brightness. This reflects the qualitative diffuse appearance determined by visual morphology and seen in Figure 4. Interestingly, the dwarf ellipticals display greater homology than the normal ellipticals. On average, dwarf ellipticals are more self-similar than normal ellipticals and can be scaled up and down in effective radius to reproduce the full range in luminosity displayed by the sample, although that range in total luminosity is much smaller than the normal elliptical sample, detailed inclusion of fainter dE's would quantify this statement.

The class of compact ellipticals (cE) also follow the normal elliptical sequence. Although it does not seem to have been noticed by previous studies, all the compact ellipticals have luminosities less than $-20 M_{g}$. Presumingly a tidal origin to this class of ellipticals would naturally produce a smaller, fainter object. Their close association with bright companions supports this hypothesis, although, statistically, faint ellipticals would be the lesser companion to a pair or small group. Thus, their smaller effective radii $\left(r_{e}\right)$ are expected for the smaller profiles per given luminosity. There is nothing peculiar about their profile shapes, although many have poorer accuracy at low surface brightness levels (due to isophotal confusion with their brighter companions) and may display undetected tidal stripping signatures in their outer isophotes. However, to within a few $r_{e}$, their profile shapes and characteristic surface brightnesses are well matched to the templates and by an extrapolation from brighter, isolated ellipticals.

\section{CONCLUSIONS}

Given the wide dispersion in kinematics for bright ellipticals, presumingly from a history of mergers (Bender et al. 1992), it is surprising that their structure is as uniform as given by Figure 2 or as indicated by template construction. Thus, a structural dichotomy between normal and dwarf ellipticals also seems odd in comparison to many numerous astronomical relationships that smoothly trace the ellipticals sequence over 12 magnitudes in luminosity. For example, color and metallicity progresses smoothly from dwarf to normal ellipticals (driven by stellar mass, as it relates to the termination of galactic winds, Poggianti et al. 2001). Even some structural parameters display no break with luminosity, such as the concentration index $n$ and central surface brightness $\left(\mu_{o}\right)$. In fact, key to the discussion of dichotomy is the combined behavior of the $n$ index with luminosity and size (Graham \& Guzmán 2003; Gavazzi et al. 2005). For the $n$ index is a powerful tool in parameterizing ellipticals and substitutes for velocity dispersion to produce a 'Photometric Plane' for ellipticals comparable to the Fundamental Plane (Graham 2002). The $n$ index also links dwarf and normal ellipticals by varying smoothly, and linearly, with luminosity. 
Over time, two schools of thought have formed with respect to the E-dE dichotomy. One school argues that dwarf and bright ellipticals represent one structural family with a gradual increase in $n$ with luminosity (Jerjen \& Binggeli 1997; Graham \& Guzmán 2003) and, presumably, a common origin scenario. The other school insists that dwarfs and bright ellipticals are structurally distinct regardless of the scaling relationships presented from Sérsic fits due to a separation seen in numerous physical properties between the two types of ellipticals (Kormendy et al. 2009; Janz \& Lisker 2009) particularly their kinematic separation in the Fundamental Plane (Bender et al. 1992). Chen et al. (2010) argues that one should ignore morphological classifications (as they are subjective) and direct our analysis to the various fitting parameters. Under this scheme, a vast majority of ellipticals follow one sequence with a small minority of objects displaying deviant structure (such as high central surface brightnesses) that could well be the result of environmental factors.

Overall, there is no strict boundary by mass, size, density or kinematics to define a dwarf elliptical from a normal elliptical (aside from an artificial luminosity division deduced from morphology), but structure seems to be the singular feature. Certainly structure, as it reflects into visual appearance, is the primary consideration that morphologists use to divide ellipticals into dwarfs and normal or compact classes. This is also true for the latetype galaxies, such as dwarf irregulars and disk galaxies, which separate by scalelength even though both types are well fit by an exponential profile (Schombert 2006). This reinforces a connection between dI's and dE's (Grebel et al. 2003) as structural differences usually signal varying formation scenarios (Driver et al. 2011) or strong merger histories to disrupt the original structural form.

In summary, the evidence presented in this study supports the dichotomy in structure between normal and dwarf ellipticals despite the wide number of nonstructural correlations that argue for a continuum between elliptical types. The evidence falls into three parts. First, the extension of the normal elliptical sequence to fainter luminosity extends to the linear relations beyond the expectations from Sérsic fits that has been interpreted as connection between normal and dwarf ellipticals. Second, many of those faint ellipticals have structural properties (such as effective surface brightness) that are well outside the range of dwarf ellipticals of the same luminosity (i.e., stellar mass). A sufficient number of these ellipticals are found over a range of environments to dismiss the conjecture that this is a small subset whose structure has been disturbed by environmental effects.

Third, and the most salient point, the template analysis clearly demonstrates that the average profiles of dwarf ellipticals are distinct from the normal elliptical sequence of profile shape with luminosity. This last result derives directly from the conclusion that both dwarf and normal ellipticals display, at least, weak homology. Bright ellipticals are clearly not self-similar (Schombert 2015), but do display an quantifiable change in profile shape with luminosity. Compact or faint ellipticals also follow the bright elliptical template sequence and are distinct from the shape of dE's. Dwarf ellipticals display stronger homology (you can roughly scale any $\mathrm{dE}$ profile upward or downward in luminosity as they are, on average, ex- ponential in shape). But, dwarf ellipticals profiles can not be scaled into normal ellipticals without significant changes to their relationship between characteristic surface brightness and radius. The two types of ellipticals appear to follow separate evolutionary histories with respect to structure. As dE's are only found in clusters, it is possible that some environmental process dominates their structural formation and evolution.

A separate structural path for dE's would argue for the parallel galaxy sequence proposed by Kormendy \& Bender (2012). In this scheme, ellipticals, S0's and gasrich disks form parallel sequences defined by formation processes and environmental secular evolution can reshape their general appearance. Structurally, dE and SO's are more closely related than normal ellipticals and dE's. This would make the dE sequence the low luminosity counterparts to the higher luminosity S0's, although their kinematics differ.

Acknowledgements:

The software and funding for this project was supported by NASA's Applied Information Systems Research (AISR) and Astrophysics Data Analysis Program (ADAP) programs. Data used for this study was based on observations made with SDSS where funding has been provided by the Alfred P. Sloan Foundation, the Participating Institutions, the National Science Foundation, the U.S. Department of Energy, the National Aeronautics and Space Administration, the Japanese Monbukagakusho, the Max Planck Society, and the Higher Education Funding Council for England, In addition, this research has made use of the NASA/IPAC Extragalactic Database (NED) which is operated by the Jet Propulsion Laboratory, California Institute of Technology, under contract with the National Aeronautics and Space Administration. 


\section{REFERENCES}

Bekki, K., Couch, W. J., Drinkwater, M. J., \& Gregg, M. D. 2001, ApJ, 557, L39

Bender, R., Burstein, D., \& Faber, S. M. 1992, ApJ, 399, 462

Binggeli, B., \& Cameron, L. M. 1991, A\&A, 252, 27

Binggeli, B., Sandage, A., \& Tarenghi, M. 1984, AJ, 89, 64

Caldwell, N. 1983, AJ, 88, 804

Cawson, M. G. M., Kibblewhite, E. J., Disney, M. J., \& Phillipps, S. 1987, MNRAS, 224, 557

Chen, C.-W., Côté, P., West, A. A., Peng, E. W., \& Ferrarese, L. 2010, ApJS, 191, 1

Chilingarian, I., Cayatte, V., Revaz, Y., et al. 2009, Science, 326, 1379

Conselice, C. J., Gallagher, J. S., III, \& Wyse, R. F. G. 2001, ApJ, 559, 791

Dabringhausen, J., \& Fellhauer, M. 2016, MNRAS, 460, 4492

Dabringhausen, J., Hilker, M., \& Kroupa, P. 2008, MNRAS, 386, 864

Driver, S. P., Hill, D. T., Kelvin, L. S., et al. 2011, MNRAS, 413, 971

Gavazzi, G., Donati, A., Cucciati, O., et al. 2005, A\&A, 430, 411

Graham, A. W., \& Driver, S. P. 2005, PASA, 22, 118

Graham, A. W., \& Guzmán, R. 2003, AJ, 125, 2936

Graham, A. W. 2002, MNRAS, 334, 859

Graham, A. W. 2013, Planets, Stars and Stellar Systems. Volume 6: Extragalactic Astronomy and Cosmology, 6, 91

Grebel, E. K., Gallagher, J. S., III, \& Harbeck, D. 2003, Astronomische Nachrichten Supplement, 324, 90

Janz, J., \& Lisker, T. 2009, Astronomische Nachrichten, 330, 948

Jedrzejewski, R. I. 1987, MNRAS, 226, 747
Jerjen, H., \& Binggeli, B. 1997, The Nature of Elliptical Galaxies; 2nd Stromlo Symposium, 116, 239

Kormendy, J., \& Bender, R. 1996, ApJ, 464, L119

Kormendy, J. 1977, ApJ, 218, 333

Kormendy, J. 2009, Galaxy Evolution: Emerging Insights and

Future Challenges, 419, 87

Kormendy, J., Fisher, D. B., Cornell, M. E., \& Bender, R. 2009, ApJS, 182, 216

Kormendy, J., \& Bender, R. 2012, ApJS, 198, 2

Lisker, T. 2009, Astronomische Nachrichten, 330, 1043

Lisker, T., Grebel, E. K., Binggeli, B., \& Glatt, K. 2007, ApJ, 660,1186

Lisker, T., Grebel, E. K., \& Binggeli, B. 2008, AJ, 135, 380

Lisker, T., Grebel, E. K., \& Binggeli, B. 2008, AJ, 135, 380

Norris, M. A., Kannappan, S. J., Forbes, D. A., et al. 2014, MNRAS, 443, 1151

Pfeffer, J., \& Baumgardt, H. 2013, MNRAS, 433, 1997

Poggianti, B. M., Bridges, T. J., Mobasher, B., et al. 2001, ApJ, 562,689

Sandage, A., \& Binggeli, B. 1984, AJ, 89, 919

Schombert, J. M. 1984, Ph.D. Thesis,

Schombert, J. M. 1987, ApJS, 64, 643

Schombert, J. M. 2006, AJ, 131, 296

Schombert, J. 2011, Astrophysics Source Code Library, ascl: 1107.011

Schombert, J. M. 2013, PASA, 30, e034

Schombert, J. M. 2015, AJ, 150, 162

Schombert, J. 2016, arXiv:1609.07500

Schombert, J. 2017, in prep

Wirth, A., \& Gallagher, J. S., III 1984, ApJ, 282, 85 